\title{
EMPORION : UN EXCEPTIONNEL GROS MODULE POSTARCHAÏQUE À LA TÊTE DE SILÈNE / CHOUETTE DANS CARRÉ CREUX
}

\author{
EMPORION: AN EXCEPTIONAL POSTARCHAIC LARGE MODULE WITH THE HEAD OF SILENUS / \\ OWL IN AN INCUSE SQUARE
}

\author{
JEAN-ALBERT CHEVILLON \\ Chercheur indépendant \\ jeanalbertchevillon@wanadoo.fr \\ http://orcid.org/0000-0003-0519-0811 \\ PERE PAU RIPOLLÈS \\ Universitat de València \\ pere.p.ripolles@uv.es \\ http://orcid.org/0000-0001-9680-6911
}

Recepción: 18-11-2016

Aceptación: 03-04-2017

\begin{abstract}
Résumé
Parmi les monnaies « lourdes » attribuables à l'Emporion grecque pour sa période archaïque nous avons publié, en 2013, un spécimen à la tête de Silène de face avec un carré creux au revers. Cet exemplaire fut mis, en tenant compte de sa valeur pondérale importante, en équivalence avec les autres nominaux recensés pour la phase A de ce monnayage. Or, suite à notre récente mise en avant d'un groupe inédit d'oboles postarchaïques empuritaines avec au revers un carré creux contenant une chouette et des lettres autour, nous proposons de relier dans le temps ces deux émissions. Nous nous appuierons pour cela, entre autres, sur la présence désormais avérée dans le carré creux de notre exemplaire d'une chouette au style et aux dimensions équivalentes, ainsi que sur une métrologie de ces deux groupes qui se complète parfaitement. Ce spécimen devient le premier représentant d'un nominal « lourd » pour cette phase.
\end{abstract}

Mots clée. Emporion; Numismatique; V siècle av. J.-C.

\begin{abstract}
Among the archaic «heavy» coinages attributed to the Greek Emporion that we published in 2013, there was a specimen with the head of Silenus on the front and an incuse square on the reverse. This coin was included, according to its heavy weight, in the archaic coinages listed for the Emporitan phase A. Now, as a result of our recent research of an unpublished group of Emporitan postarchaic obols with an incuse square containing an owl
\end{abstract}

and letters around, we suggest that these two denominations are related. We base our proposal, among other reasons, on the presence of an owl engraved, with a similar style and equal dimensions, inside the incuse square. The metrology of these two groups does also perfectly complement each other. This coin becomes the first representative specimen of a heavy denomination minted in Emporion during the postarchaic phase.

Key words. Emporion; Numismatics; 5th century BC.

\section{Resumen}

Entre las monedas «pesadas» atribuidas a la fase arcaica de Emporion, que publicamos en el año 2013, se encontraba una pieza con cabeza de Sileno de frente en anverso y cuadrado incuso en el reverso. Este ejemplar fue atribuido al grupo de emisiones de la fase arcaica debido a que su elevado peso se correspondía con los nominales de la fase A. No obstante, como consecuencia del estudio de un grupo inédito de óbolos post-arcaicos emporitanos, que muestra un mochuelo rodeado de letras griegas dentro de un cuadrado incuso, ahora proponemos relacionar ambos tipos monetarios. Para ello nos basamos en la identificación de un mochuelo en el interior del cuadrado incuso de nuestra moneda, con un estilo y dimensiones equivalentes, así como en la metrología de estos dos grupos que se complementan perfectamente. Esta moneda se convierte en la primera pieza perteneciente a un nominal pesado de la fase post-arcaica.

Palabras clave. Emporion; Numismática; siglo V a.C. 


\section{LA MONNAIE}

Présentée dans notre travail de synthèse sur le monnayage archaïque de l'Emporion grecque, qui couvre la production monétaire émise entre les années 515480 av. J.-C., (Ripollès y Chevillon, 2013: phase A groupe 2.1) elle a été aussi reproduite et commentée dans une autre étude (Chevillon y Ripollès, 2014b: 282, no 5) (Fig. 1); elle fut décrite avec, à l'avers, une tête de Silène de face et au revers un carré creux. Poids 4,2 g, 15,2-12 mm. Figueres (Girona). Origine: sud de la Catalogne.

Néanmoins, à la lumière des dernières découvertes relatives à la phase postarchaïque du monnayage empuritain (ca. 480-440 a.C.), en particulier avec notre travail sur les oboles aux protomés «adossées» avec les têtes retournées d'un taureau et d'un lion et, au revers, une chouette avec quelques lettres autour dans un carré creux (Fig. 2) (Chevillon y Ripollès, 2014a: 41-46), et après un examen visuel du tétrobole à la tête de Silène de face, la chronologie et la dénomination de ce type monétaire se devaient d'être reconsidérées ${ }^{1}$. En premier lieu, une observation précise du tétrobole a permis de révéler la présence, à l'intérieur du carré creux du revers ${ }^{2}$, d'une chouette de style équivalent à celles des oboles aux protomés «adossées». Orienté à gauche, avec une tête au fort volume, l'animal présente une forme et un traitement qui s'accordent parfaitement avec ceux des oboles précitées (Fig. 3).

Leur style de gravure, assez «faible», fait partie des constantes qui caractérisent la production monétaire pour la période postarchaïque (ca.480-440 a.C. $)^{3}$. Cette

1. Un des sujets de ce travail est la correction de quelques éléments en relation avec la caractérisation de cette pièce. En tenant compte de son revers à carré creux, elle fut incluse dans la production archaïque d'Emporion. (ca. 515-480 av. J.-C., voir une courte vision de cette période dans Chevillon et Ripollès, 2014b: 281), mais l'examen de cette monnaie a permis de reconsidérer sa description et sa chronologie. D'abord, la monnaie ne pesait pas 4,2 g, mais 3,68 g. Sa dénomination en tant que pentobole n'est donc plus correcte et elle doit être désormais considérée comme un tétrobole. Il en va de même pour le lieu de trouvaille qui fut donné au départ pour le sud de la Catalogne et qui, au final, provient de la commune de Figueres.

2. Seulement étudié au départ par le biais d'une photo, il nous a fallu attendre ces derniers mois pour avoir la possibilité d'examiner plus précisément ce spécimen. Nos vifs remerciements vont à Gabriel Villaronga qui nous a largement aidé dans cette recherche.

3. La phase postarchaïque, qui débute à Emporion vers 480 av. J.-C., se singularise par l'apparition des doubles types (p. ex. $A C I P 3,15,54)$ qui restent, encore pour quelques temps, mélangés avec des séries à motif unique (p. ex. ACIP 1,2) ainsi que par la présence des premières légendes (le plus souvent limitées à quelques lettres). Elle correspond également à l'arrêt définitif du type « figé » à la tête de bélier de la phase $\mathrm{B}$ archaïque et marque le retour aux frappes à types multiples qui caractérisent la phase $\mathrm{A}$ (sur ce thème: Chevillon, Ripollès y López, 2013: 10-14).
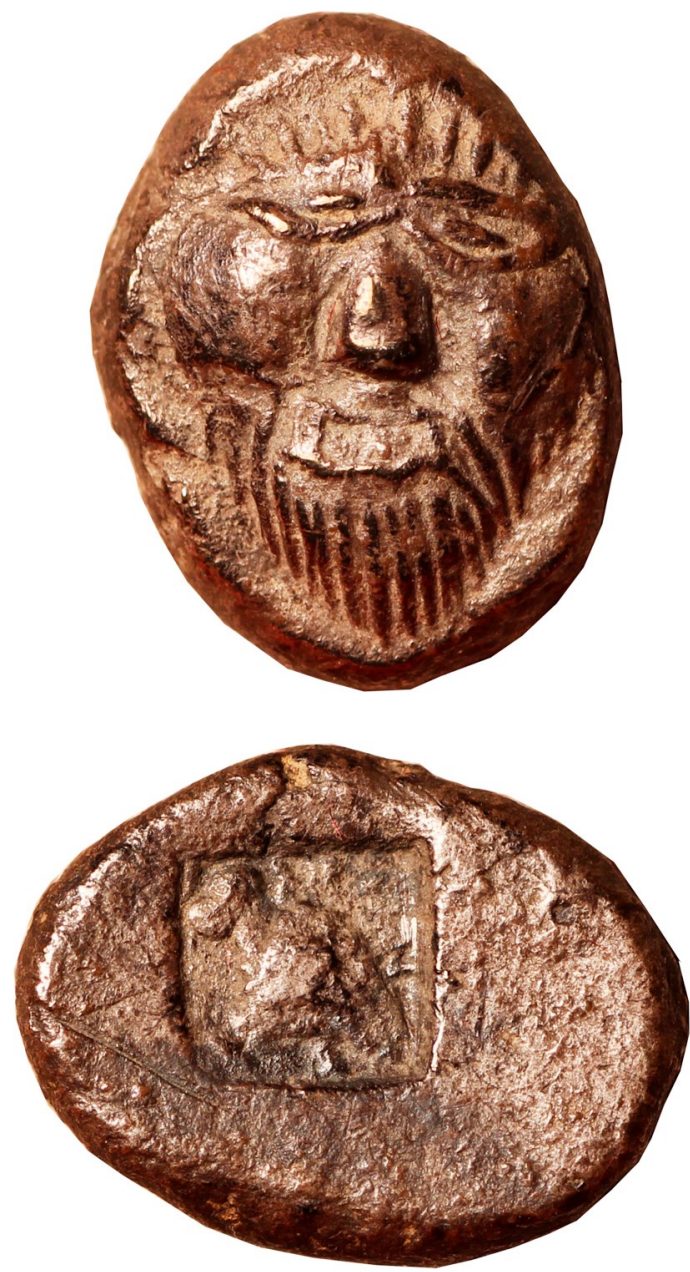

Figure 1: Tétrobole d'Emporion avec une tête de Silène de face à l'avers. Phase postarchaïque (vers 480 - 440 av. J.-C.).

spécificité est également confirmée pour les motifs de droit de ces deux émissions. Autre élément, la forme du revers de notre spécimen qui, comme pour les oboles «à la chouette» s'avère à fond plat et à coins ronds. Il en va de même pour les dimensions du carré creux de notre gros module à la tête de Silène qui sont équivalentes à celles des carrés de nos oboles (toutes entre 5 et $6 \mathrm{~mm}$ ). Ce lien, particulièrement signifiant, confirme, un peu plus, la «contemporanéité» de ces monnaies. Il semble signaler que l'émetteur n'a pas jugé nécessaire de graver un coin à taille adaptée sur la série « lourde» en préférant s'en tenir à un revers de l'obole.

En second lieu, l'examen direct de ce tétrobole nous a permis de corriger le poids qui nous avait été communiqué initialement. À la suite d'une nouvelle pesée, il s'avère que la masse réelle de cet exemplaire s'élève à $3,68 \mathrm{~g}$, et non 4,20 g comme signalé initialement. Ce nouvel élément nous amène à faire un lien étroit entre les oboles postarchaïques (poids théorique de $0,92 \mathrm{~g}$ ) (Chevillon, 2011: 185-187) et notre spécimen dont la valeur pondérale correspond parfaitement à celle d'un tétrobole (poids théorique de $3,68 \mathrm{~g}$ ). Le choix de cette obole de 0,92 g - dans l'étalon phocaïco-persique fut adopté par Emporion vers 480 av. J.-C. (Chevillon 

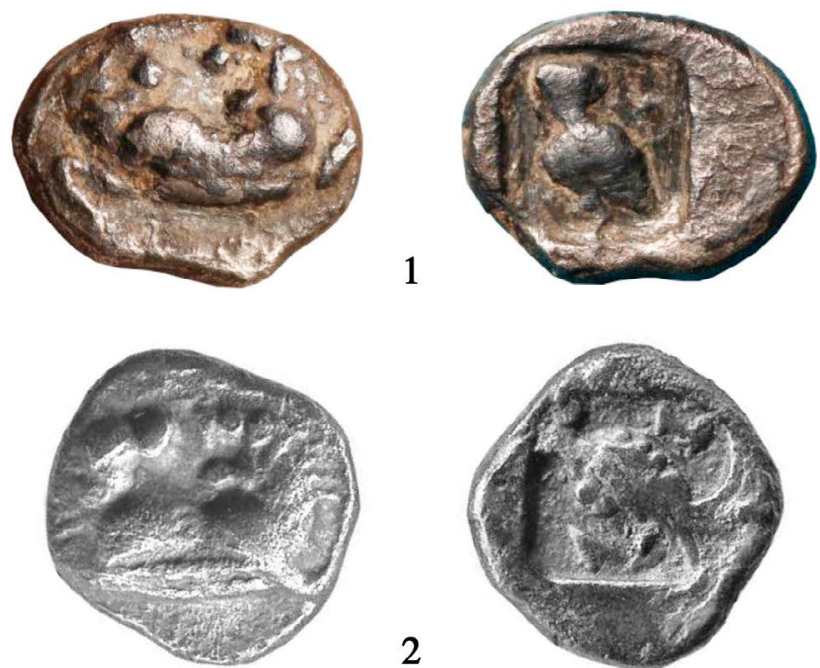

2
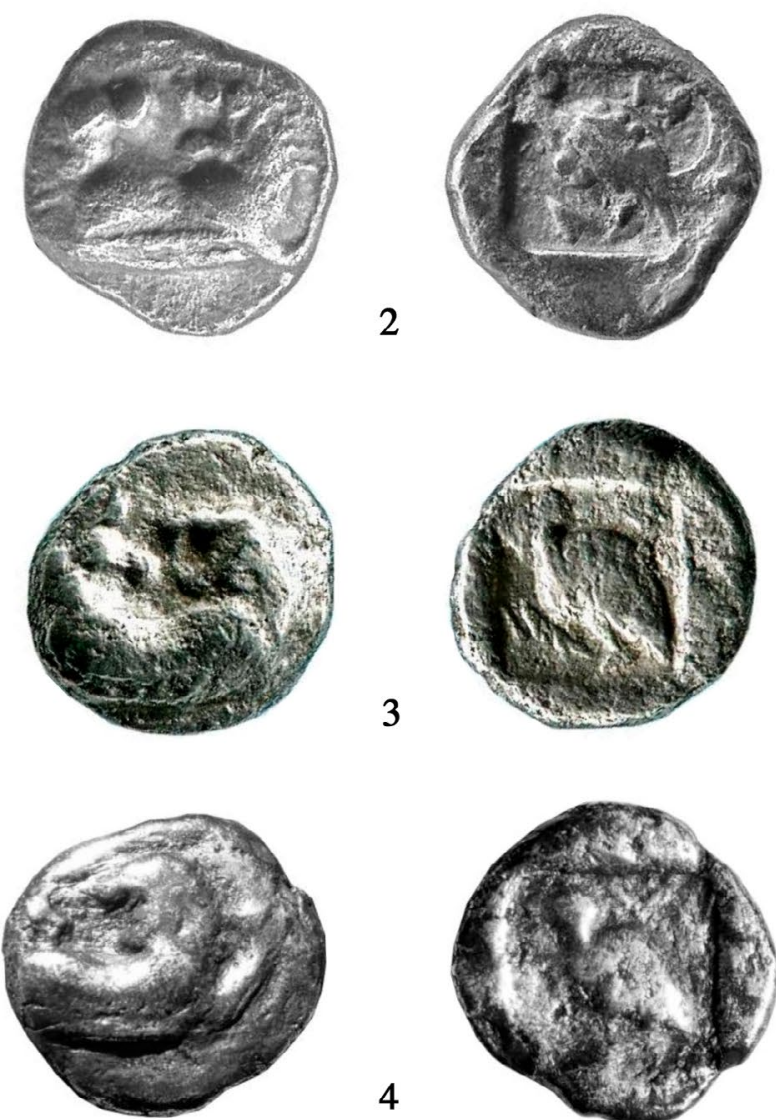

4
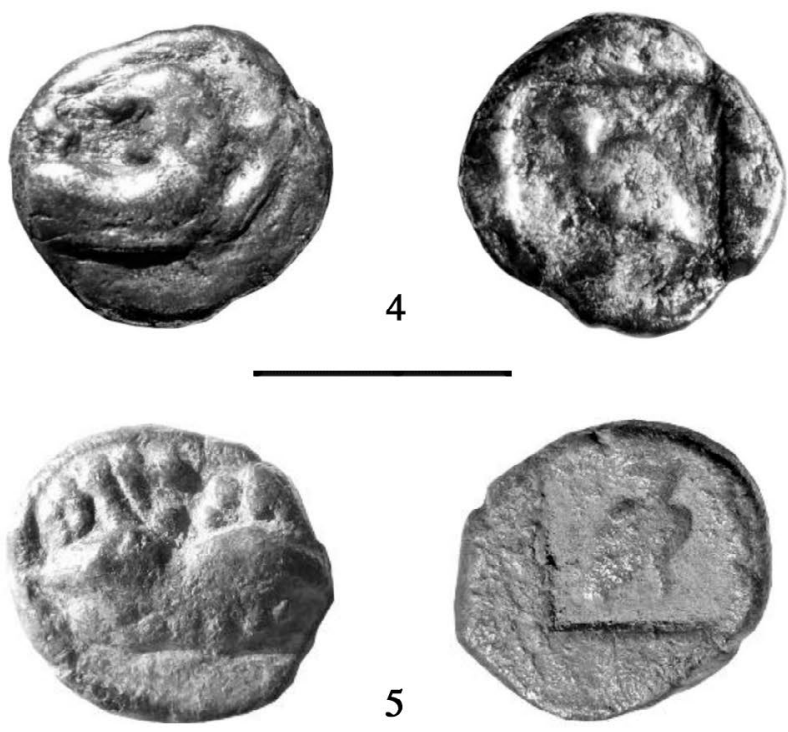

5
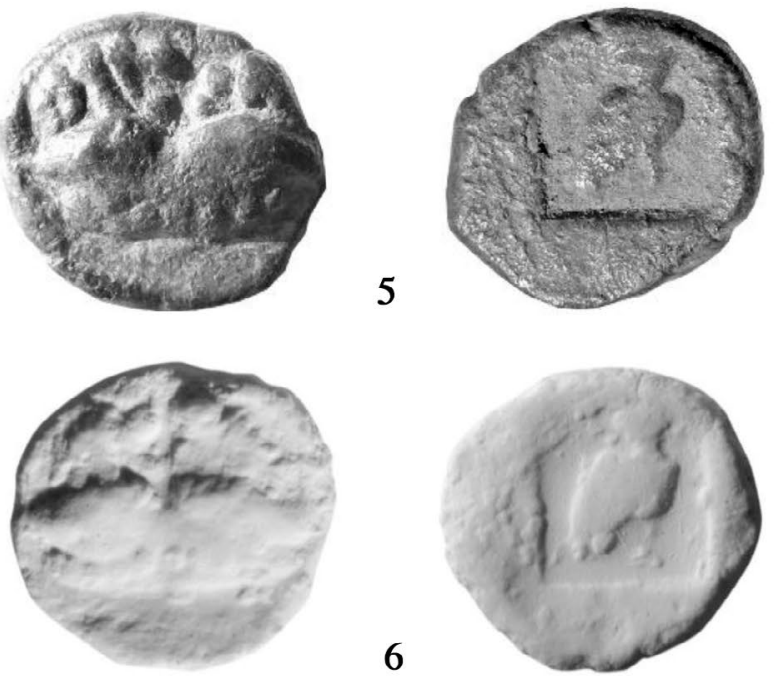

Figure 2: Oboles d'Emporion avec les têtes retournées d'un taureau et d'un lion. Phase postarchaïque (vers 480 - vers 440 av. J.-C.). 1: $0,92 \mathrm{~g}, 7,5-10 \mathrm{~mm}$, coll. privée (Espagne). Provenance: entre Fortià et Arenys d'Empordà (près de Roses et d'Empúries); 2: 0,79 g, 8,7-9,8 mm, coll. privée (France); 3: 0,75 g, 8,3-8,7 mm, coll. privée (France); 4: 0,60 g, 8-8,7 m, coll. privée (France); 5: 1,136 g, 9 mm. Coll. privée (Espagne). Provenance: Vilamalla dans l'Alt Empordà proche d'Emporion; 6: 0,87 g, 8,5 mm, coll. S. Vela (Sagonte). Provenance: Camp de Morvedre (Sagonte). 

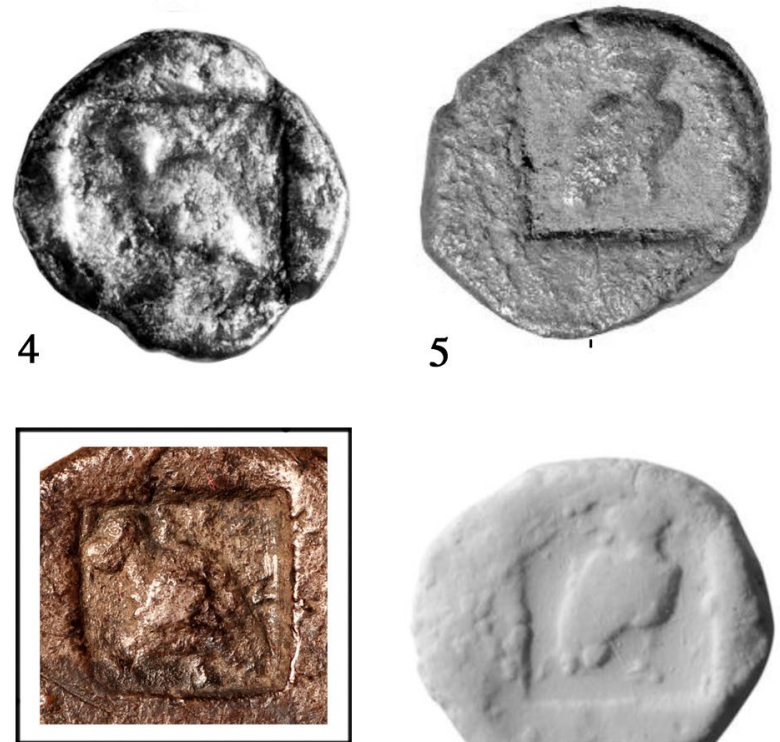

Tétrobole
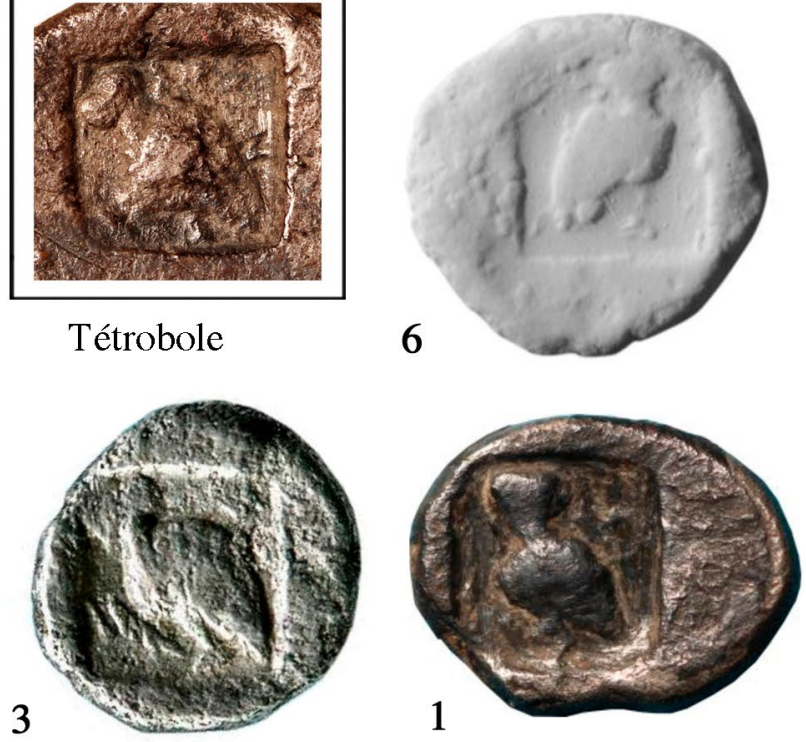

Figure 3: Comparaison entre les revers à la chouette du tétrobole et des oboles de la phase postarchaïque (pour le détail des pièces illustrées ici se référer aux données de la Fig. 2).

y Ripollès, 2014a: 45). Il caractérise l'ensemble des frappes postarchaïques de l'atelier. Il est intéressant de noter que Massalia va également adopter, presque aussitôt, pour sa phase postarchaïque, une obole de masse équivalente (Chevillon, 2013: 135-158). Nous datons globalement la production postarchaïque d'Emporion des années 480 - 440 av. J.-C.

\section{COMMENTAIRE}

Ainsi, une relation précise a pu être établie entre ces rares monnaies à la typologie très originale: protomés «adossées» avec les têtes retournées d'un taureau et d'un lion / chouette avec légende autour dans un carré creux, et une monnaie de poids lourd $(3,68 \mathrm{~g})$ présentant un revers identique ${ }^{4}$. Ce spécimen, dont

4. Un deuxième spécimen à la tête de silène de face (Ripollès y Chevillon, 2013: phase A - groupe 3.1) reste classé parmi les groupes d'hémidrachmes de cette période. Avec une masse de 2,86 g, un style de gravure différent et un revers en svastika, cet exemplaire ne s'apparente pas à notre tétrobole. la valeur pondérale correspond à celle d'un tétrobole dans l'étalon en cours à Emporion et à Massalia à la même époque, pouvait donc être échangé contre quatre oboles postarchaïques. L'intérêt de ce tétrobole à la tête de Silène de face repose, en particulier, sur le fait que nous n'avons pas, à ce jour, de trace de nominaux de poids élevé à la fois dans la production empuritaine mais également massaliète ${ }^{5}$ pour leurs phases postarchaïques respectives qui n'étaient documentées, jusqu'à présent, que par des oboles, hémioboles et tétartémoria ${ }^{6}$. Nul doute, que d'autres trouvailles, pour une période qui reste encore largement à explorer, nous permettront d'apporter des éléments nouveaux sur ce premier groupe postarchaïque de poids « lourd » pour l'atelier monétaire d'Emporion.

\section{RÉFÉRENCES}

$A C I P=$ Villaronga, L. y Benages, J. (2011). Les Monedes de l'Edat Antiga a la Península Ibèrica. Barcelona.

Chevillon, J.-A. (2011). Emporion: un groupe inédit à la tête de Dionysos. En Barter, Money and Coinage in the Ancient Mediterranean (10th-1st centuries BC)». Actas del IV Encuentro Peninsular de Numismatica Antigua (EPNA) (pp. 185-187). Anejos de AespA LVIII. Madrid: Consejo Superior de Investigaciones Científicas.

Chevillon, J.-A. (2013). La phase postarchaïque du monnayage de Massalia. Revue Numismatique, 169, 135-158. DOI: https://doi.org/10.3406/numi.2012.3178

Chevillon, J.-A. y Ripollès, P. P. (2014a). The Greek far west: an exceptional adaptation of a design from Asia Minor with bull and lion foreparts. Journal of the Numismatic Australian Association, 25, 41-46.

Chevillon,J.-A.y Ripollès, P.P.(2014b). Emporion archaïque: genèse d'un revers-type. Lucentum, XXXIII, 281-285. DOI: https://doi.org/10.14198/LVCENTVM2014.33.18

Chevillon, J.-A., Ripollès, P. P. y López, C. (2013). Les têtes de taureau dans le monnayage empuritain du Ve s. av. J.-C. OMNI, revue numismatique, 6, 10-14.

Ripollès, P. P. y Chevillon, J.-A. (2013). The Archaic Coinage of Emporion. Numismatic Chronicle, 173, 1-21. Tiré de : http://www.jstor.org/stable/43859722

5. À Massalia, la phase postarchaïque débute vers 475 av. J.$\mathrm{C}$, alors qu'à Théliné la production s'interrompt définitivement à ce moment-là. Nous avons montré que la phase postarchaïque empuritaine a débuté un peu avant (vers 480 av. J.-C.).

6. Ces dénominations, comme toutes celles qui ont été frappées au cours de la phase archaïque, se caractérisent par un certain relâchement métrologique lors de l'ajustement des flans, ce qui crée des problèmes pour identifier certaines dénominations. Pour les émissions postarchaïques, le pied utilisé repose sur la base théorique d'une obole de $0,92 \mathrm{~g}$, mais dans les divers groupes il est possible de trouver des poids moyens décalés d'environ cinq centièmes de gramme. 